\title{
Scaling the Advantages of Intra-channel Nonlinearity Compensation in Future Flexible Optical Networks
}

\author{
Danish Rafique ${ }^{(1)}$ and Andrew D. Ellis \\ Photonic Systems Group, Tyndall National Institute, and University College Cork, Prospect Row, Cork, \\ Ireland. ${ }^{(1)}$ was with Tyndall National Institute and University College Cork. danishrafique@hotmail.com
}

\begin{abstract}
We report that, contrary to common perception, intra-channel nonlinearity compensation offers significant improvements of up to $4 \mathrm{~dB}$, in nonlinear tolerance (Q-factor), in a flexible traffic scenario, and further improvements with increasing local link dispersion, for an optical transport network employing flexible 28Gbaud PM-mQAM transponders.
\end{abstract}

\section{Introduction}

While advanced modulation formats are spectrally efficient, generally they have reduced tolerance towards channel impairments [1-2]. One of the promising ways to alleviate such effects is the use of digital signal processing (DSP) employing linear and nonlinear compensation using digital back-propagation (DBP) [3]. Typically, wide bandwidth DBP is considered to be nonviable due to high computational load and limitations on electronics [4], and single channel DBP (intra-channel nonlinear compensation) has been shown to only enable modest improvements between $\sim 1$ $2 \mathrm{~dB}$, with respect to linear compensation only [5-6]. However, this has only been investigated for systems employing homogenous traffic, where all the channels carry same power. As network upgrades are carried out, it is likely that channels employing different multi-level formats will become operational, and the network traffic will become inhomogeneous [7-9]. It is thus important to determine if intra-channel nonlinear compensation is worth the extra computational effort in future flexible networks.

Another approach to improve nonlinear tolerance is the use of fibres with high local dispersion coefficients. It is well-understood that such a choice decreases the dispersion length, leading to reduced inter-channel nonlinearities, however at the expense of timing jitter and ghost pulses appearing within a channel [10]. This leads to important questions, such as how much local link dispersion may be tolerated with linear compensation, and the performance benefits of intra-channel nonlinearity compensation.

In this paper we focus on quantifying the benefits available from intra-channel nonlinearity compensation, for a network designed with aforementioned scenarios in mind. We demonstrate that for inhomogeneous networks with unequal-power multi-level co-propagating traffic, intra-channel nonlinear compensation enables up to $4 \mathrm{~dB}$ improvement in nonlinear tolerance, irrespective of the co-propagating modulation format, compared to the expected $\sim 1.5 \mathrm{~dB}$ improvements for equal-power transmission (same launch power for all the channels). We further demonstrate that the benefits of intra-channel nonlinear compensation are enhanced by increasing the local link dispersion such that for a potential near-future inhomogeneous network, $5.5 \mathrm{~dB}$ relative improvements are available if intrachannel nonlinear compensation is deployed, instead of linear compensation only.

\section{Transmission Setup}

Fig. 1 illustrates the simulation setup. The transmission system comprised fifteen WDM channels employing 28Gbaud PM-mQAM $(m=4$, 16) at a channel spacing of $50 \mathrm{GHz}$. The central channel was always 28Gbaud PM-16QAM (at $1550 \mathrm{~nm}$ ), and the neighbours were selected to be PM-mQAM channels (unless mentioned otherwise). For all the carriers both the polarisation states were independently modulated using de-correlated $2^{15}$ and $2^{16}$ pseudo-random bit sequences (PRBS), for $x$ and $y$ - polarisation states, respectively. The optical transmitters consisted of continuous wave lasers $(5 \mathrm{kHz}$ line-width) followed by two nested Mach-Zehnder Modulator structures for $\mathrm{x}$ - and y polarisation states. The 28Gbaud PMmQAM signals were propagated over an uncompensated transmission link with $80 \mathrm{~km}$ spans, and erbium doped fibre amplifiers. The fibre had attenuation of $0.2 \mathrm{~dB} / \mathrm{km}$, nonlinearity coefficient of $1.5 / \mathrm{W} / \mathrm{km}$, and dispersion coefficient of $20 \mathrm{ps} / \mathrm{nm} / \mathrm{km}$ (unless specified otherwise). Each amplifier was modelled by assuming a $4.5 \mathrm{~dB}$ noise figure and $16 \mathrm{~dB}$ gain.

After fibre transmission, the central PM-16QAM signal was coherently-detected to give the baseband signal sampled at 2samples/symbol followed by digital field reconstruction from the in-phase and quadrature samples. Transmission impairments were digitally compensated in two scenarios. Firstly, by using linear compensation (LC) alone (the back-propagation section in Fig. 1 was by-passed), employing finite impulse response filters adapted using a least mean square algorithm. In the second case, electronic compensation was applied via single-channel DBP (SC-DBP), which was numerically 


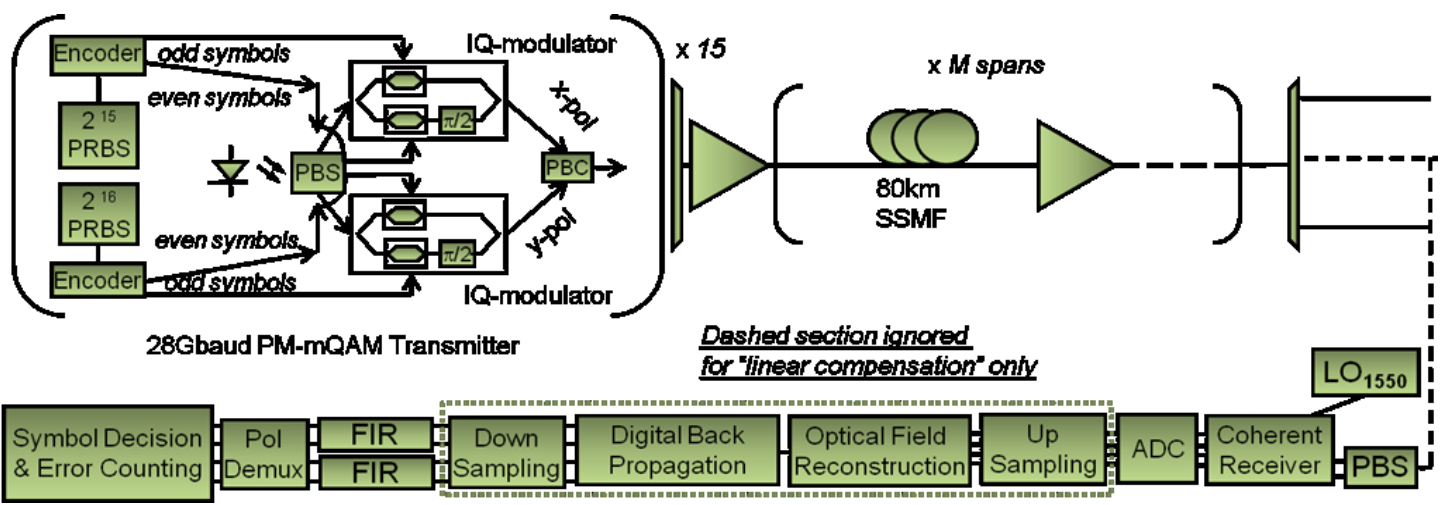

Fig. 1: Simulation setup for 28Gbaud WDM PM-mQAM transmission.

implemented by up-sampling the received signal to 16 samples/symbol and reconstructing the optical field from the in-phase and quadrature samples, followed by split-step Fourier method based solution of nonlinear Schrödinger equation. Polarisation de-multiplexing and residual dispersion compensation was then performed using a standard butterfly structure, followed by carrier phase recovery. Finally, the symbol decisions were made, and the performance assessed by direct error counting (converted into $Q$-factor, $Q^{2}$ in $d B$ ). All the numerical simulations were carried out using VPItransmissionMaker®V8.6, and the DSP was performed in MATLAB®V7.10.

\section{Results and discussions}

Typical results of our simulations are shown in Fig. 2 as a function of signal launch power $\left(P_{L}\right)$ for the central PM-16QAM channel. The channel power allocation in a flexible network is an important system design choice [7-8], consequently, $P_{L}$ of all the PM-mQAM neighbours was fixed at the near-optimal power for heterogeneous transmission [8] (unless specified otherwise). Specifically, we show four heterogeneous transmission scenarios: Circles, Squares and Stars, PM-4QAM/PM-16QAM/PM64QAM neighbours, respectively: $P_{L}=0 \mathrm{dBm}$, Diamonds, PM-4QAM neighbours: Degraded $\mathrm{P}_{\mathrm{L}}$ $=4 \mathrm{dBm}$. As it can be seen, with low-power lower-order neighbouring traffic (circles), most likely network scenario, SC-DBP enables $4 \mathrm{~dB}$ improvement in Q-factor and $6 \mathrm{~dB}$ in optimal $\mathrm{P}_{\mathrm{L}}$ (or nonlinear threshold, NLT) due to effectively reduced inter-channel nonlinear effects. Also, one may observe similar improvements with PM-16QAM/PM-64QAM neighbours (squares and stars), which further confirm the advantages of SC-DBP even in the presence of homogenous or higher-order traffic. The independence of test-channel on neighbouring modulation format may be attributed to saturated peak-to-average power, after few kms, for multi-order QAM. If $P_{L}$ of neighbouring traffic is intentionally degraded, or the cross-channel effects are exacerbated, diamonds, one may still observe an improvement of $1 \mathrm{~dB}$ in $\mathrm{Q}$-factor and up to $5 \mathrm{~dB}$ improvements in NLT. This suggests that even if the network-controlled launch is not in favour of the propagating traffic, benefits may be ascertained from SC-DBP. Also, for the sake of completeness, we plot the performance of test-channel with equal-power (PM-16QAM neighbours: $P_{L}$ optimized with test channel), lines, where one may see conventional improvement of $\sim 1.5 \mathrm{~dB}$.

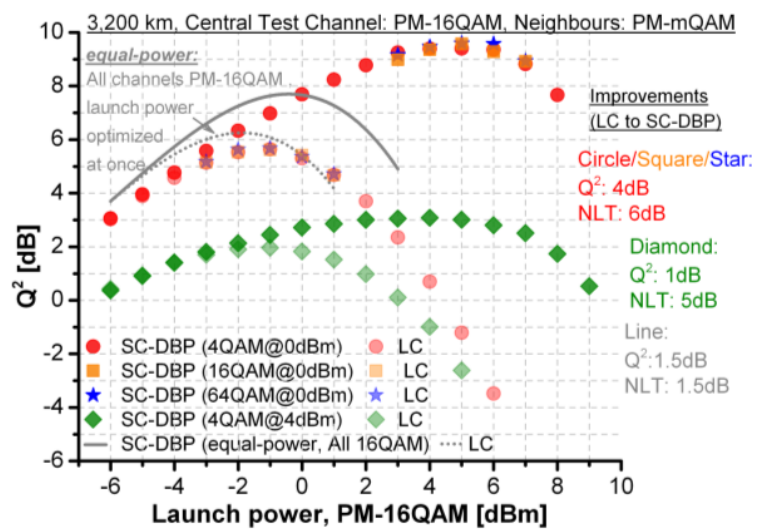

Fig. 2: $Q^{2}$ of central $P M-16 Q A M$ test-channel as a function of launch power of PM-16QAM test-channel. Circles, Squares, Stars: PM-4QAM/PM-16QAM/PM-64QAM neighbours at $0 \mathrm{dBm}$, Diamonds: PM-4QAM neighbours at 4dBm, Lines: curve fits PM-16QAM neighbours with launch power optimized with test-channel. Dark symbols and solid line: SC-DBP, Light symbols and dashed line: LC.

Having established the available benefits from SC-DBP in a flexible network, we explore fibre design characteristics in a practical inhomogeneous network scenario (PM-16QAM co-propagating with PM-4QAM channels at $0 \mathrm{dBm})$, to further improve nonlinear tolerance, in context of intra-channel nonlinear compensation. Fig. 3 plots the performance of PM-16QAM test-channel as a function of local link dispersion coefficient, both after LC (light symbols) and SC-DBP (dark symbols). It can be seen that increasing the absolute magnitude of local dispersion enables improvements in system performance, with even greater 


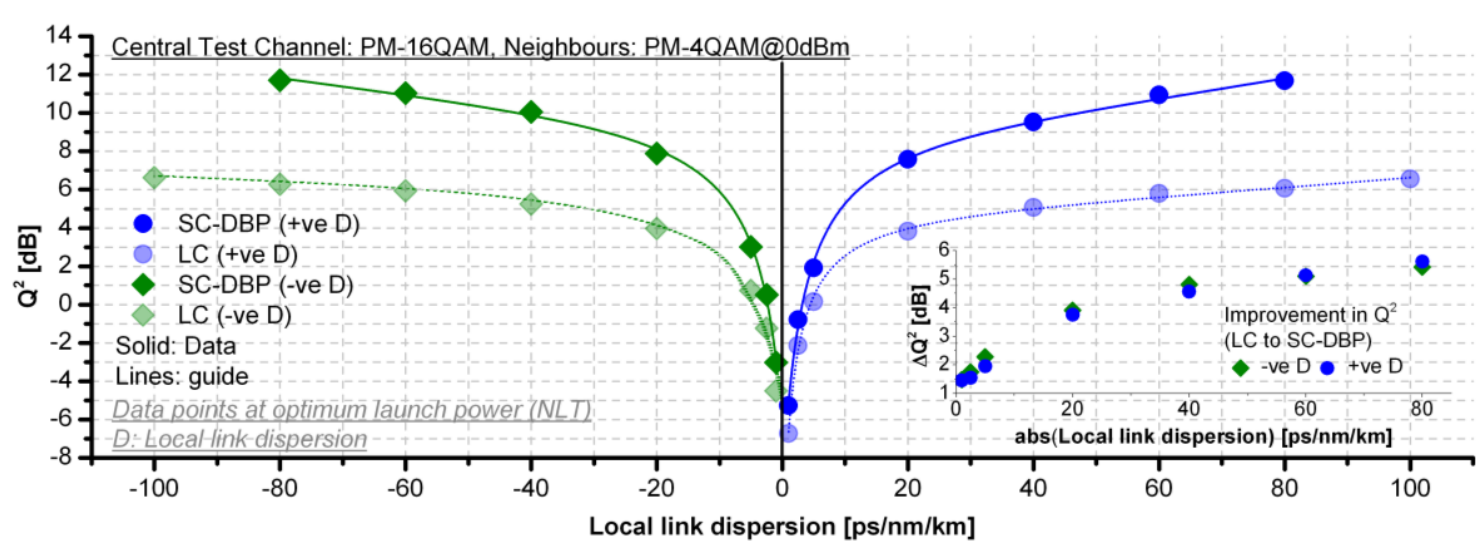

Fig. 3: $Q^{2}$ of central PM-16QAM test-channel versus local link dispersion. Circles: Positive dispersion coefficient, Diamonds: Negative dispersion coefficient. Dark symbols: SC-DBP, Light symbols: LC, Lines: guide. Inset shows relative improvement from LC to SC-DBP for negative/positive dispersion coefficient.

improvements after SC-DBP. In particular, after LC, initially the performance improves aggressively, due to reduced phase-matching effects with increasing local link dispersion. As the local dispersion is increased further, the improvement margin reduces due to the tradeoff between inter-channel nonlinearities (favouring high local dispersion to increase channel walk-off), and intra-channel nonlinearities (favouring low local dispersion to minimize peak-to-average power ratio). Nonetheless at $80 \mathrm{ps} / \mathrm{nm} / \mathrm{km}$ of local dispersion (both \pm coefficients), more than $2 \mathrm{~dB}$ improvements are observed, compared to conventional single-mode fibre $(17 \mathrm{ps} / \mathrm{nm} / \mathrm{km})$.

Likewise, significantly greater benefits are enabled by SC-DBP with increasing local link dispersion, as shown in Fig. 3. This can be attributed to increased intra-channel nonlinear effects with high local dispersion, which may be essentially compensated using SC-DBP, while also reducing phase-matching, leading to reduced inter-channel nonlinearities. In particular, with a local dispersion of $\pm 80 \mathrm{ps} / \mathrm{nm} / \mathrm{km}, \mathrm{Q}$-factor may be improved by $3.5 \mathrm{~dB}$, compared to that of single-mode fibre $(17 \mathrm{ps} / \mathrm{nm} / \mathrm{km})$, hinting that already available negative dispersion fibres (with slightly higher loss than $0.2 \mathrm{~dB} / \mathrm{km}$ ) may be deployed with a sufficient margin. The inset shows similar improvements when LC is replaced with SCDBP, with up to $5.5 \mathrm{~dB}$ improvements in Q-factor at $\pm 80 \mathrm{ps} / \mathrm{nm} / \mathrm{km}$ of local dispersion. Note that the performance improvements are largely consistent with analytical predictions of [1], except in the very-low dispersion (inter-channel parametric process dominate due to extreme phase-matching) or very-high dispersion (intrachannel parametric process dominate due to rapid pulse spread) regimes. One may argue that such a high dispersion coefficient may result in increased complexity of SC-DBP.
However, recently proposed correlated DBP [11] may be effectively employed to take dispersion into account, with significant complexity reductions. Also, pre-dispersed spectral inversion has been demonstrated which may again offset any complexity associated with digital signal processing [12].

\section{Conclusions}

We have demonstrated that advantages of intrachannel nonlinear compensation are significantly beyond conventionally thought bound of $\sim 1-2 \mathrm{~dB}$. In particular, we have shown that in a flexible network scenario, intra-channel nonlinearity compensation may improve the nonlinear tolerance by $4 \mathrm{~dB}$, irrespective of modulation order of the co-propagating traffic. Furthermore, we have shown that increasing the local link dispersion enables significant performance improvements. We have reported up to $2 \mathrm{~dB}$ and $3.5 \mathrm{~dB}$ improvements with linear compensation, and nonlinear compensation, respectively, compared to the typical dispersion coefficient of $17 \mathrm{ps} / \mathrm{nm} / \mathrm{km}$, and a relative improvement between linear and nonlinear compensation of about $5.5 \mathrm{~dB}$.

This work was supported by Science Foundation Ireland: Grant numbers 06/IN/I969 and 10/CE/I1853.

We also thank N.J. Doran for useful discussions.

\section{References}

[1] A.D. Ellis, et al., JLT, 28, 423 (2010)

[2] D.Rafique, et al., Optics Express, 19, 5219 (2011)

[3] X.Li, et al., Optics Express, 16, 880 (2008)

[4] E.Ip, JLT, 28, 939 (2010)

[5] S.J Savory, et al., PTL, 22, 673 (2010)

[6] X. Zhou, et al., OFC, NthB4 (2011)

[7] T. Wuth, et al., OFC, NTuB3 (2008)

[8] D.Rafique, et al., PTL, 23, 1213 (2011)

[9] A.Nag, et al., JLT, 28, 466 (2010)

[10] R.-J.Essiambre, et al., Optical Fibre

Telecommunications IV B. Academic (2002).

[11] L. Lei, et al., OFC, OWW3 (2011)

[12] D.Rafique, et al., OFC, OM3A.1 (2012) 